\title{
Enforcement of the ban on aristolochic acids in Chinese traditional herbal preparations on the Dutch market
}

\author{
Martijn J. Martena $\cdot$ Jacqueline C. A. van der Wielen • \\ Leo F. J. van de Laak • Erik J. M. Konings • \\ Henk N. de Groot • Ivonne M. C. M. Rietjens
}

Received: 31 January 2007 /Revised: 11 April 2007 / Accepted: 12 April 2007 / Published online: 8 May 2007

(C) Springer-Verlag 2007

\begin{abstract}
In traditional chinese medicine several Aristolochia species are used. Aristolochia spp. contain a mixture of aristolochic acids (AAs), mainly AA I and AA II which are nephrotoxicants and carcinogens. After AA-related nephropathy (AAN) and urothelial cancer were described in female patients in Belgium following intake of AA-contaminated herbal preparations, herbs with AAs were prohibited worldwide. Confusing nomenclature can cause AA contamination of certain Chinese traditional herbal preparations (THPs). Here we report the results of investigations by the Dutch Food and Consumer Product Safety Authority (VWA) into the presence of AAs in THPs sampled on the Dutch market using a liquid-chromatography--mass spectrometry method. Between 2002 and 2006 we sampled 190 Chinese THPs using recent information on Chinese THPs potentially containing AAs. AA I was found in 25 samples up to a concentration of $1,676 \mathrm{mg} / \mathrm{kg}$. AA II was also found in 13 of these samples up to $444 \mathrm{mg} / \mathrm{kg}$. All 25 positive samples including Mu Tong, Fang Ji, Tian Xian Teng and Xi Xin were part of a group of 68 THPs identified as possibly containing AAs. In a worst-case scenario, use of a sample of Mu Tong with the highest AA content over a 7-day period would result in the same intake levels of AAs which
\end{abstract}

M. J. Martena $(\varangle) \cdot$ J. C. A. van der Wielen •

L. F. J. van de Laak • E. J. M. Konings · H. N. de Groot

Food and Consumer Product Safety Authority (VWA),

Region South,

P.O. Box 2168, 5600 CD Eindhoven,

The Netherlands

e-mail: martijn.martena@vwa.nl

I. M. C. M. Rietjens

Division of Toxicology, Wageningen University,

Tuinlaan 5,

6703 HE Wageningen,

The Netherlands significantly raised the cancer risk in the Belgian AAN cases. Our results show that contaminated THPs still can be found on the market following worldwide publicity. Therefore, it can be concluded that testing of possibly AAcontaminated THPs is still essential.

Keywords Chinese herb nephropathy · Aristolochic acid . Mu Tong · Traditional chinese medicine $\cdot$ Herbal remedies
Abbreviations
AA Aristolochic acid
AAN Aristolochic acid nephropathy
BEN Balkan endemic nephropathy
CHN Chinese herb nephropathy
CYP Cytochrome P450
LC Liquid chromatography
LOD Limit of detection
LOQ Limit of quantification
MS Mass spectrometry
QC Quality control
SCM Standardized control material
TCM Traditional chinese medicine
THP Traditional herbal preparation
VWA Dutch Food and Consumer Product Safety Authority

\section{Introduction}

In traditional chinese medicine (TCM) Aristolochia species such as $A$. fangchi and A. manshuriensis and others are used to treat snake and insect bites, promote lactation or urination and reduce edema [1]. Aristolochia spp. are used for medicinal purposes worldwide. Many herbs from the genus Aristolochia and several species of the genus 
Asarum, both belonging to the family of the Aristolochaceae, contain several aristolochic acids (AAs) often accompanied by aristolactams [2-4]. Literature on the toxicity of the Aristolochiaceae and related analytical papers mostly focus on a naturally occurring mixture of AAs mainly consisting of AA I and AA II (Fig. 1) [5].

AAs were shown to be nephrotoxic and carcinogenic in animal studies with rodents $[5,6]$. It has been shown in rat studies that the renal proximal tubule is an important target of AA toxicity which can result in renal failure $[7,8]$. AAs have been suggested to play a role in the Balkan endemic nephropathy (BEN) characterized by renal interstitial fibrosis. Seeds of A. clematitis, which is ubiquitous in BEN-affected areas, may have contaminated grain [9]. The International Agency for Research on Cancer concluded that herbal preparations with Aristolochia spp. are carcinogenic to humans and that naturally occurring mixtures of AAs are probably human carcinogens as well [10]. In humans the hepatic and renal activation of AAs is attributed to reductive metabolic activation by cytochrome $\mathrm{P} 450$ (CYP) 1A1, CYP1A2, NAD(P)H:quinone oxidoreductase and others. The resulting ultimate carcinogenic species is able to form adducts with DNA, which in turn can cause mutations and neoplastic changes $[5,11]$. We recently reviewed the toxic action of AA in some detail [12].

TCM is gaining popularity in Western countries but certain safety issues of Chinese traditional herbal preparations (THPs) such as the deliberate use of high amounts of heavy metals [13] and the presence of AAs invariably require attention. In the USA and the Netherlands Chinese THPs are regarded as foods. In Dutch food law THPs are regulated as herbal preparations in the Commodities Act "Herbal preparations." Since it came into force in early 2001, this Act has prohibited the presence of AAs and their derivatives in herbal preparations with Aristolochia spp. This ban was recently extended to all herbal preparations irrespective of the plant species present. Several other countries, such as the UK, the USA, Canada, Australia and New Zeeland, have since 2000 significantly limited or prohibited the sale of AA-containing herbs and issued warnings [14-18]. These measures were inspired by a

Fig. 1 Structure of aristolochic acid $\mathrm{I}\left(\mathrm{R}\right.$ is $\left.\mathrm{OCH}_{3}\right)$ and aristolochic acid II ( $\mathrm{R}$ is $\mathrm{H})$ steadily expanding insight into the nature of the causative agent of poisonings with Chinese THPs in Belgium in the early 1990s. In 1992 a cluster of nine similar cases of renal interstitial fibrosis in female patients was identified in Brussels. All these patients were treated between 1990 and 1992 in a slimming clinic with a regimen consisting of a diet, injections and capsules containing pharmaceuticals such as fenfluramine, herbal preparations and a pancreas extract. In early 1990 the clinic had altered the THP formulation by introducing the Chinese herbs Magnolia officinalis and Stephania tetrandra to the capsules, replacing other herbs. It was suggested that $S$. tetrandra was inadvertently replaced by $A$. fangchi. Thin-layer chromatography detection of AAs in these herbal preparations failed however [19]. Afterwards AAs were found in 11 of the 12 batches of $S$. tetrandra powder delivered to Belgian pharmacies during the treatment period [20]. The disease became known as Chinese herb nephropathy (CHN). After the initial report more than 100 cases of rapidly progressing renal fibrosis associated with exposure to AAs were identified in Belgium and approximately 170 cases of AAassociated $\mathrm{CHN}$ were described in other European countries, the USA and in Asia [5]. In renal tissue of 39 patients who were treated with the Belgian slimming regimen and who were followed for $\mathrm{CHN}$-related end-stage renal failure 18 cases of urothelial cancer were identified. All tissue samples examined contained AA-related DNA adducts [21]. It was found that a total intake of more than $200 \mathrm{~g} \mathrm{~S}$. tetrandra (probably mostly replaced by $A$. fangchi) was associated with a higher risk of urothelial carcinoma [21]. A statistical analysis of the prescriptions and medical files of $71 \mathrm{CHN}$ patients showed that of all administered drugs only the cumulative dose of the contaminated $S$. tetrandra preparation could predict the renal failure progression rate [22]. The typical chronic interstitial lesions of $\mathrm{CHN}$ were reproduced in rats injected with $10 \mathrm{mg} / \mathrm{kg} /$ day of a mixture of $40 \%$ AA I and $60 \%$ AA II for 35 days [7]. Nowadays many authors prefer to use the more accurate term "aristolochic acid nephropathy" (AAN) over the term CHN.

As a result of the Belgian CHN cases it became better known why certain Chinese THPs are contaminated with AAs and what the effects of exposure to AAs can amount to. Confusion over the Chinese common name "Fang Ji," which can refer both to the roots of $S$. tetrandra and $A$. fangchi, could have caused the contamination with AAs $[19,20]$. In the trade of Chinese herbs the substitution of one plant species for another is established practice [23]. Besides the THPs known to contain Aristolochia species some THPs derived from certain plant species can be identified which can be replaced by Aristolochia species in practice. Several international food and medicine authorities have published lists of THPs suspected to contain AA. The Dutch Food and Consumer Product Safety Authority 
(VWA) has implemented these lists in its sampling strategies of Chinese THPs and analyzed AAs in commercial samples. Reports providing quantitative data on AA levels of commercial THPs possibly containing AAs are scarce. Such data could, however, be useful to validate the existing lists of suspected THPs and could help authorities to pinpoint their efforts to protect the consumer against exposure to AAs. In the present paper our results in this field over the past 4 years are presented and compared with recent scientific and regulatory data.

\section{Materials and methods}

\section{Sampling}

VWA inspectors sampled 190 Chinese THPs on the Dutch market in the period from November 2002 to June 2006. Samples were selected by using a list of single-herb THPs and multi-ingredient THPs probably and possibly containing AAs which was based on a list compiled by the FDA in 2001 [24]. The FDA list was supplemented and regularly updated in-house with data on the nomenclature of AAcontaining herbal material from books on TCM, commercial TCM databases on the Internet, warnings of other inspection agencies and literature [1, 15, 23-28, 38, 40, 42]. Table 1 presents an extract of our sampling list defining the most pertinent Aristolochia species in use in TCM and several plant species with which they could be exchanged. The Chinese common names in the table refer to plants and the parts used and are given in Pin Yin, which is a phonetic representation of Chinese characters. The corresponding Latin pharmaceutical name also presented is a combination of the part of the plant and often its binomial botanical name. Both types of nomenclature are seen in the market. Table 2 presents examples of formulas which can be potentially contaminated with AAs through the presence of Mu Tong, Fang Ji or Xi Xin. For sampling, products were selected by formula name or by screening the ingredient list for suspected herbs. Along with THPs known or suspected to contain AAs, THPs were sampled at random as well. THP names were copied as labeled and are presented in this paper without alterations. Generally no authentication of the herb was performed.

THPs were collected in TCM stores, oriental food stores, wholesale dealers, importers or TCM practitioners throughout the Netherlands. Sampling inspections were held at least each year. Locations were selected from the VWAinspection database. During inspections of these locations, new suppliers and stores were also identified and visited. THPs were sampled in prepackaged form in capsules or tablets or in many cases in the form of coarse herbal material from glass containers sometimes with limited or no labeling. Samples were taken on the basis of quantities supplied to consumers, which is one unit (e.g., bottle, package or container) or in case of coarse herbal material in amounts higher than $10 \mathrm{~g}$. Mixtures of the coarse materials are assembled in TCM shops according to a formula prescribed by an in-house TCM practitioner. This THP mixture is then prepared at home as a decoction for which

Table 1 Examples of single-herb traditional herbal preparations (THPs) possibly containing aristolochic acids

\begin{tabular}{llll}
\hline Pin Yin name & Part used & Botanical name & Latin pharmaceutical name, including alternative names \\
\hline THPs with Aristolochia species & & \\
Guang Fang Ji & Root & Aristolochia fangchi & Radix Aristolochia fangchi/Aristolochiae Fangchi \\
Ma Dou Ling & Fruit & A. contorta and A. debilis & Fructus Aristolochiae \\
Tian Xian Teng & Herb & A. contorta and A. debilis & Herba Aristolochiae/Caulis Aristolochiae \\
Guan Mu Tong & Stem & A. manshuriensis & Caulis Aristolochia manshuriensis/Aristolochiae \\
& & & Manshuriensis \\
Qing Mu Xiang & Root & A. debilis & Radix Aristolochiae \\
Xun Gu Feng, Bai Mao & Herb & A. mollissima & Herba Aristolochiae Mollissimae \\
Teng & & & \\
THPs possibly contaminated & & \\
Han Fang Ji & Root & Stephania tetrandra & Radix Stephania tetrandra \\
Mu Fang Ji & Root & Cocculus trilobus and C. orbiculatus & Radix Cocculi Trilobi/Radix Cocculus trilobus/Radix Cocculi \\
Chuan Mu Tong & Stem & Clematis armandii & Caulis Clematis armandii/Clematidis armandii \\
Chuan Mu Tong & Stem & Clematis montana & Caulis Clematidis/Clematis montana/Clematis armandii \\
Bai Mu Tong & Stem & Akebia quinata & Caulis Akebia quinata \\
Bai Mu Tong & Stem & Akebia trifoliata & Caulis Akebia trifoliata \\
Bai Mao Teng, Bai Ying & Herb & Solanum lyratum & Herba Solani Lyrati \\
\hline
\end{tabular}

From $[1,23,24,27,42]$ 
Table 2 Examples of multi-ingredient THPs possibly containing AAs

Formula name in Pin Yin, in alphabetical order

\begin{tabular}{lll}
\hline Formulas with Mu Tong or Fang Ji & & \\
Anyang Jingzhi Gao & Fang Ji Huang Qi Tang & Quell Fire \\
Ba Zheng Wan & Fenqing Wulin Wan & Shang Zhong Xia Tong Yong Tong Feng Fang \\
Chi Kuan Yen Wan & Fu Ke Fen Qing Wan & Shi Xiang Fan Shen Wan \\
Chu Shi Wei Ling Tang & Gan Lu Xiao Du Dan & Shu Feng Huo Xue Tang \\
Chun Yang Zheng Ji Wan & Guan Xin Su He Wan & Shu Jing Huo Xue Tang \\
Da Huang Qing Wei Wan & Guo Qi Yin & Tienchi Hugu Wan \\
Da Qiang Huo Tang & Ji Jiao Li Huang Wan & Xiao'er Jindan tablets \\
Dang Gui Si Ni Tang & Ji Sheng Ju He Wan & Xiao Feng San \\
Dang Gui Si Ni Wan & Jia Wei Wu Lin San & Xiao Huo Luo Dan \\
Dao Chi San & Ju He Wan & Xiao Xu Ming Tang \\
Dao Chi Wan & Kat Kit Wan & Xin Yi Wan \\
Dieda Wan & Kuanhsin Suhowan & Xuan Bi Tang \\
Er Jia Jian Zheng Qi San & Long Dan Xie Gan Tang & Zhisou Huatan Wan \\
Ershiwuwei Songshi Wan & Long Dan Xie Gan Wan & Zhu Ling Tang \\
Fang Ji Fu Ling Tang & Mu Fang Ji Tang & \\
Formulas with Xi Xin & & \\
Chuan Xiong Cha Tiao San & Du Huo Ji Sheng Tang & San Bi Tang \\
Chuan Xiong Cha Tiao Wan & Jiu Wei Qiang Hou Tang & She Gan Ma Huang Tang \\
Da Huang Fu Zi Tang & Ling Gan Wu Wei Jiang Xin Tang & Tong Guan San \\
Da Qin Jiao Tang & Ma Huang Fu Zi Xi Xin Tang & Wu Mei Wan \\
Dang Gui Si Ni Tang & Qu Feng Zhi Bao Dan & Xiao Qing Long Tang \\
\hline
\end{tabular}

From $[15,24,25,27,38,40,42]$

the herbs are boiled in water or other liquids. The strained liquid is then consumed [1].

\section{Experimental}

The method used to quantify AA I and AA II was based on the method described by Flurer et al. [29]. The entire sample was homogenized or in the case of capsules the contents of all the capsules were taken and homogenized, and from this a laboratory sample was taken for further analysis. After homogenization $25 \mathrm{ml}$ of extraction solution was added to $1 \mathrm{~g}$ of sample. The extraction solution existed of $80 \%$ methanol, $18 \%$ water and $2 \%$ formic acid. The samples were shaken for $90 \mathrm{~min}$ in a shaking machine (Gerhardt LS-20, position 9). Then they were allowed to precipitate for about $1 \mathrm{~h}$. An aliquot of $1 \mathrm{ml}$ was then centrifuged at a minimum of $10,000 \mathrm{~g}$. The supernatant was transferred to a vial and hermetically sealed for liquid chromatography (LC)-mass spectrometry (MS) analysis. No concentration step was needed.

An ion-trap LC-MS system from Thermofinnigan (LCQ Advantage) equipped with a quaternary pump, an autosampler with a column oven, a photodiode array detector and an integration system together with LC-MS software was used for analysis. The separation was performed on an Alltima C-18 column $(150 \mathrm{~mm} \times 3.2-\mathrm{mm}$ inner diameter-
5- $\mu \mathrm{m}$ particle size) with an Alltima $\mathrm{C}-18$ precolumn (7.5 $\mathrm{mm} \times 3.0-\mathrm{mm}$ inner diameter $-5-\mu \mathrm{m}$ particle size) using gradient elution. LC conditions are listed in Table 3. The MS detection was performed by electrospray ionization using the positive mode. The MS conditions are listed in

Table 3 Liquid chromatography $(L C)$ conditions for the determination of AA I and AA II

\begin{tabular}{|c|c|c|c|}
\hline Parameters & \multicolumn{3}{|l|}{ Conditions } \\
\hline Analytical column & \multicolumn{3}{|c|}{$\begin{array}{l}\text { Alltima } \mathrm{C}-18 \text { column }(150 \mathrm{~mm} \times 3.2-\mathrm{mm} \text { inner } \\
\text { diameter }-5 \text { - } \mu \mathrm{m} \text { particle size })\end{array}$} \\
\hline Precolumn & \multicolumn{3}{|c|}{$\begin{array}{l}\text { Alltima } \mathrm{C}-18(7.5 \mathrm{~mm} \times 3.0-\mathrm{mm} \text { inner } \\
\text { diameter }-5-\mu \mathrm{m} \text { particle size })\end{array}$} \\
\hline Column temperature & \multicolumn{3}{|c|}{$30^{\circ} \mathrm{C}$} \\
\hline Injection volume & \multicolumn{3}{|c|}{$20 \mu \mathrm{l}$ (full-loop injection) } \\
\hline Flow rate & \multicolumn{3}{|l|}{$0.30 \mathrm{ml} / \mathrm{min}$} \\
\hline Mobile phase & \multicolumn{3}{|c|}{$\begin{array}{l}\text { Eluent } \mathrm{A}: 10 \mathrm{mmol} \text { ammonium formate in } \\
1 \% \text { formic acid } \\
\text { Eluent B: Methanol }\end{array}$} \\
\hline \multirow[t]{8}{*}{ LC gradient } & Time (min) & Eluent A $(\%)$ & Eluent B (\%) \\
\hline & 0.00 & 50 & 50 \\
\hline & 10.00 & 20 & 80 \\
\hline & 21.00 & 20 & 80 \\
\hline & 22.00 & 0 & 100 \\
\hline & 25.00 & 0 & 100 \\
\hline & 26.00 & 50 & 50 \\
\hline & 34.00 & 50 & 50 \\
\hline
\end{tabular}


Table 4. Before injection of samples, the system was equilibrated using 50\% eluent $\mathrm{A}$ and $50 \%$ eluent $\mathrm{B}$. Quantification of AA I and AA II was based on a standard mixture obtained from Sigma-Aldrich (Zwijndrecht, The Netherlands) containing 43\% AA I and 54\% AA II.

For every series of samples several quality checks were performed, including a check of the validity of calibration, a check on the ratio of the first to the second daughter ion and the analysis of quality control (QC) samples (two standardized control materials, SCMs, containing AA I or AA II). For this QC material the mean and a 95\% confidence interval were established. Each measurement of this QC material had to comply with this $95 \%$ confidence interval. AA was only quantified when all quality checks for a series of samples were in line with the desired performance characteristics.

\section{Results and discussion}

Characteristics of the analytical method

For the in-house validation of the method for quantifying AA I and AA II, the limit of detection (LOD) and the limit of quantification (LOQ) were determined. The LOD was defined as the concentration which is 3 times higher than the range of the chromatographic background of the second daughter ion. The LOD determined in this way was $1.0 \mathrm{mg} / \mathrm{kg}$ for AA I and $1.6 \mathrm{mg} / \mathrm{kg}$ for AA II. The LOQ was defined as twice the LOD and was $2.0 \mathrm{mg} / \mathrm{kg}$ for AA I and $3.2 \mathrm{mg} / \mathrm{kg}$ for AA II. Two calibration curves were used, both of which were linear with correlation coefficients of at least 0.97 . The linearity for the low-level AA ranges was determined between 86 and $430 \mathrm{ng} / \mathrm{ml}$ for AA I and between 108 and $540 \mathrm{ng} / \mathrm{ml}$ for

Table 4 Mass spectrometry (MS) conditions for the determination of AA I and AA II

\begin{tabular}{ll}
\hline Parameters & Conditions \\
\hline Capillary temperature & $250^{\circ} \mathrm{C}$ \\
Sheath gas & $45 \%$ \\
Aux/sweep & 10 \\
Source voltage & $5.00 \mathrm{kV}$ \\
Source current & $80.00 \mu \mathrm{A}$ \\
Capillary voltage & $9.00 \mathrm{~V}$ \\
Tube lens offset & $15.00 \mathrm{~V}$ \\
Scan & $\mathrm{m} / \mathrm{z} 100.00-400.00$ \\
Parent ion AA I & $\mathrm{m} / \mathrm{z} 359.0:$ first daughter ion $\mathrm{m} / \mathrm{z} 298.0 ;$ \\
& $\mathrm{second}$ daughter ion $\mathrm{m} / \mathrm{z} 341.7$ \\
Parent ion AA II & $\mathrm{m} / \mathrm{z} 329.1$ : first daughter ion $\mathrm{m} / \mathrm{z} 267.9 ;$ \\
& $\mathrm{second}$ daughter ion $\mathrm{m} / \mathrm{z} 294.0$ \\
\hline
\end{tabular}

AA II. The range of application for preparations containing high levels of AAs was determined as 430-6,450 ng/ml for AA I and as $540-8,100 \mathrm{ng} / \mathrm{ml}$ for AA II. The calibration curves for the high concentration range were established by quadratic regression. Preparations containing even higher amounts of AAs were diluted with the extraction solution. The validity of the calibration curve, retention times and LOD were checked with each series of samples. Quantification of both AA I and AA II was based on the first daughter ions; however, amounts were only quantified when the relative intensity of the second daughter ion in proportion to the first daughter ion was within specific limits. For this the peak surface of the second daughter ion was calculated as a percentage of the peak surface of the first daughter ion. For AA I the relative intensity of the second daughter ion $(\mathrm{m} / \mathrm{z} 341.7)$ had to be $44.0 \pm 11.0 \%$ of the first daughter ion $(\mathrm{m} / \mathrm{z} 298.0)$. Similarly for AA II the relative intensity of the second daughter ion $(m / z 294.0)$ had to be $53.5 \pm 10.7 \%$ of the first daughter ion $(\mathrm{m} / \mathrm{z} 267.9)$.

For the recovery studies a blank THP sampled from the market (labeled as $\mathrm{Mu}$ Tong and Clematis armandii and analytically shown to be free of AA I and AA II) was spiked at levels between 2 and $11 \mathrm{mg} / \mathrm{kg}$. As Mu Tong samples were expected to be frequently contaminated, this blank Mu Tong sample without AAs was considered to be a relevant model for our research and the recovery studies were therefore performed on this sample. The recoveries determined from six replicate measurements under repeatability conditions and in the range $2-11 \mathrm{mg} / \mathrm{kg}$ were 87 and $82 \%$, respectively, for AAI and AAII with relative standard deviations of 3.7 and $3.5 \%$, respectively. The reproducibility was defined as 2.8 times the standard deviation obtained from results determined by different operators and at different times using SCMs. For AA I a SCM, a Xi Xin THP sampled from the market containing $4.75 \mathrm{mg} / \mathrm{kg}$ AA I, was used to determine the reproducibility, which amounted to $0.94 \mathrm{mg} / \mathrm{kg}$. For AA II a SCM was prepared from a mixture of a Guan $\mathrm{Mu}$ Tong THP sampled from the market (an Aristolochia sp. with a high level of AA II) and the blank $\mathrm{Mu}$ Tong sample without any AAs which was used for the recovery studies. This mixture contained $3.76 \mathrm{mg} / \mathrm{kg}$ AA II and the reproducibility was $0.47 \mathrm{mg} /$ $\mathrm{kg}$. Both SCMs were analyzed in each series. These performance characteristics of the method are in agreement with results reported by Trujillo et al. [30].

Confusion of herbs in TCM and sampling of AA-containing THPs

Besides a sensitive analytical method, an effective sampling protocol needed to be developed for enforcement of the ban on AAs. Central to this protocol was information on which Chinese herbs can possibly be replaced by herbs that 
contain AAs. In TCM confusion of herbs occurs frequently and can result from similarities in appearance, mistakes in (ancient) textbooks, counterfeits and in many cases ambiguous nomenclature [31]. Contamination of THPs with AAs can often be traced back to confusion over nomenclature. Common or vernacular names of plants are, as opposed to binomial botanical names, not very reliable for unambiguous identification of the particular species as, for instance, the interpretation of common names can even differ between geographical regions. In TCM several plant species share a Chinese common name with an Aristolochia sp. and this common name could be seen as a group name for the species concerned. When a prefix is added to the group name the common name refers to only one or two plant species of the group; in many cases, however, only the group name is used. The prefix can point at a region where the plant is grown; for example, the prefix "Chuan" refers to the Sichuan province [1]. The common name Fang Ji refers to at least four plant species but in combination with the prefix "Guang" it is exclusively used for the root of $A$. fangchi. There is also the possibility that a herb has more than one common name, which can lead to confusion as well. For instance, it was recently reported that A. mollissima is not only called Xun Gu Feng but Bai Mao Teng as well. This last common name is also used for Solanum lyratum, which confusingly has an alternative name as well, namely, Bai Ying [32]. Substitution of $S$. lyratum by $A$. mollissima can occur when only the common name Bai Mao Teng is used when the THP is prescribed, self-medicated, traded, etc. Such a case has been reported in Hong Kong recently where a 60 -year-old man was diagnosed with renal failure and urethral cancer after he had erroneously been using Herba Aristolochiae Mollissimae instead of the desired Solanum species [31]. The authors indicated that confusion keeps recurring between the names Xun Gu Feng, Bai Mao Teng and Bai Ying [31]. Although A. mollissima is entered in the FDA list, the plant's common name is not mentioned nor is $S$. lyratum [24]. Information of this nature might prove valuable when sampling THPs for AA analysis. Tables 1 and 2 present an extract of our sampling list defining the most pertinent single-herb THPs derived from Aristolochia spp. in use in TCM, several plant species which could be replaced by Aristolochia spp. and a list of possibly contaminated formulas. This list proved useful for sampling 190 Chinese THPs on the Dutch market in the period from November 2002 to June 2006.

Analytical results

The Chinese common names and Latin pharmaceutical names of all 190 THPs were examined for indications that AA-containing herbs might be present. We identified 68 THPs as products which could possibly contain AAs and this subgroup contained all 25 positive samples. The analytical results of the 68 potentially AA-containing THPs are presented in Table 5 grouped by the Chinese common name. AA I was found in all 25 samples positive for AA, the AA I level of four THPs was below the LOQ and AA I contents of the remaining samples ranged between 2 and $1,676 \mathrm{mg} / \mathrm{kg}$. Together with AA I, AA II was detected in 13 samples, with the AA II content of one sample below the LOQ and that of the other samples between 4 and $444 \mathrm{mg} / \mathrm{kg}$. When THPs contained more than the relevant LOQ action was taken to remove the products from the market.

In three of five samples of single-herb THPs (Guan Mu Tong ${ }^{(2 x)}$, Qing Mu Xian, Guang Fang Ji and Tian Xian Teng) labeled with names exclusively referring to Aristolochia species, significant amounts of AA I were found ranging between 74 and more than $1,000 \mathrm{mg} / \mathrm{kg}$, implying that these samples indeed contained Aristolochia spp. The remaining two of these Aristolochia samples surprisingly contained no detectable levels of AAs. The identity of the samples was generally not authenticated however. In 11 of 12 samples of THPs with herbs from the genus Asarum, which also belongs to the Aristolochiaceae and in which AAs can be expected, low levels of AAs were detected. The remaining 11 positive samples were THPs that contained herbs which can be substituted by Aristolochia spp. and belonged to the Fang Ji and $\mathrm{Mu}$ Tong groups of Table 5. Of these, four products were incorrectly labeled with names that identified the herbs as AA-free counterparts of Aristolochia species. This shows that the problem in TCM of substitution of innocuous herbs with Aristolochia spp. is not yet resolved. The problem of substitution of Chinese herbs with toxic counterparts is not limited to Aristolochia spp. In 2001 the Dutch Health authorities, including the VWA, were faced with more than 60 poisonings with symptoms including epileptic seizures owing to consumption of a herbal tea where the spice Chinese star anise (Illicium verum) was replaced by an unidentified Illicium sp. imported from China and which was shown to contain the neurotoxin anisatin [33].

We found no AAs in THPs which to our knowledge were not likely to be contaminated with AAs and could therefore be considered as randomly sampled. These THPs are presented in Table 6 . Though AAs were absent, some of these products could pose a health risk to the user because they may contain other natural toxins. For example, we sampled two THPs labeled as Chuan Wu and Radix Aconiti Carmichaeli, which is the root of Aconitum carmichaeli. This plant and the related $A$. kusnezoffii (Cao Wu) are used in TCM for the treatment of musculoskeletal disorders and contain the potent neurotoxin and cardiotoxin aconitine. In Hong Kong cases of herb-induced aconitine poisonings are treated almost every year [34]. Also several herbs were sampled that are known to contain pyrrolizidine alkaloids (PAs), which are mutagenic and carcinogenic hepatotox- 
Table 5 LC-MS detection of AAs in Chinese THPs potentially containing AAs sampled on the Dutch market

\begin{tabular}{|c|c|c|c|c|c|c|c|c|}
\hline \multicolumn{2}{|c|}{ Chinese common name } & \multirow{2}{*}{$\begin{array}{l}\text { Latin pharmaceutical name } \\
\text { When labeled }\end{array}$} & \multicolumn{2}{|l|}{$N$} & \multicolumn{4}{|c|}{ AA $(\mathrm{mg} / \mathrm{kg})$ in positive samples } \\
\hline Group & $\begin{array}{l}\text { Specific names, } \\
\text { when labeled }\end{array}$ & & $\begin{array}{l}\text { Without } \\
\text { AA }\end{array}$ & $\begin{array}{l}\text { With } \\
\text { AA }\end{array}$ & AA I & $\begin{array}{l}\text { Mean } \\
\text { AA I }\end{array}$ & AA II & $\begin{array}{l}\text { Mean } \\
\text { AA II }\end{array}$ \\
\hline \multirow[t]{6}{*}{ Mu Tong } & Guang Mu Tong & - & & 2 & $\begin{array}{l}919 \\
>1,000\end{array}$ & & $\begin{array}{l}82 \\
>100\end{array}$ & \\
\hline & Guang $\mathrm{Mu}$ tong & Caulis Clematidis Armandii & 1 & & ND & & ND & \\
\hline & Mu Tong & - & & 1 & 1,453 & & 303 & \\
\hline & Mu Tong $(2 \mathrm{x}) /{ }_{-}^{(3 \mathrm{x})}$ & $\begin{array}{l}\text { Caulis Clematidis Armandii- } \\
\text { Akebiae/Caulis Clemat(id)is } \\
\text { Armandii }{ }^{(4 \mathrm{x})}\end{array}$ & 5 & & ND & & ND & \\
\hline & Mu Tong & Caulis Akebiae & & 1 & 1,281 & & 394 & \\
\hline & $\begin{array}{l}\text { Chung Mu Tong/ } \\
\text { Chuan Mu Tong } \\
\text { Chuang } \mathrm{Mu} \text { Tong }\end{array}$ & Caulis Akebiae ${ }^{(4 x)}$ & 1 & 3 & $\begin{array}{l}19 \\
1,113 \\
1,676\end{array}$ & 936 & $\begin{array}{l}4 \\
190 \\
444\end{array}$ & 212 \\
\hline \multirow[t]{5}{*}{$\begin{array}{l}\text { Mu tong (in } \\
\text { formula) }\end{array}$} & $\begin{array}{l}\text { Lon Dan Xie Gan (Pian)/ } \\
\text { Long Dan Xie Gan Tang }\end{array}$ & $-/$ Gentiana form & 4 & 3 & $\begin{array}{c}41 \\
49 \\
59\end{array}$ & 50 & $\begin{array}{l}11 ; \\
9 ; 14\end{array}$ & 11 \\
\hline & - & Quell Fire & 1 & & ND & & ND & \\
\hline & Xao Feng San & Tangkuei \& Arctium Formula & 1 & & ND & & ND & \\
\hline & Dao Chi San & Rehm. Clematis Armandi Form. & 1 & & ND & & ND & \\
\hline & Xiao Feng San & Tangkuei \& Arctium Formula & 1 & & ND & & ND & \\
\hline \multirow[t]{4}{*}{ Mu Xiang } & Qing Mu Xiang & $\begin{array}{l}\text { Radix Aristolochiae, "Duitse } \\
\text { pijp" }\end{array}$ & 1 & & ND & & ND & \\
\hline & Mu Xian & - & 1 & & ND & & ND & \\
\hline & Mu Xiang & Vladimiria & 1 & & ND & & ND & \\
\hline & Guang Mu Xiang & - & 1 & & ND & & ND & \\
\hline $\begin{array}{l}\text { Tian Xian } \\
\text { Teng }\end{array}$ & Tian Xian Teng & Caulis Aristolochiae & & 1 & 74 & & 33 & \\
\hline \multirow[t]{5}{*}{ Fang Ji } & Niu Ru Shi & Cocculi Sarm. Rad. & & 1 & $<\mathrm{LOQ}$ & & ND & \\
\hline & Guang Fang Ji & - & 1 & & ND & & ND & \\
\hline & Fang Ji & - & 1 & & ND & & ND & \\
\hline & Fanji & Sclerotium Poriae Cocos & & 1 & 12 & & $<$ LOQ & \\
\hline & Fang Ji/- & Radix Stefaniae Tetrandiae ${ }^{(2 x)}$ & 2 & & ND & & ND & \\
\hline \multirow{2}{*}{$\begin{array}{l}\text { Fang Ji (in } \\
\text { formula) }\end{array}$} & Fang Ji Qi Tang & Wutian \& Astrag. Comb & 1 & & ND & & ND & \\
\hline & Mu Fang Ji Tang & $\begin{array}{l}\text { Gypsum, Cinn \& Ginseng } \\
\text { Comb }\end{array}$ & & 1 & 524 & & 21 & \\
\hline Xi Xin & Xi Xin $(\mathrm{Bei})^{(1 \mathrm{x})} / \mathrm{Xi} \mathrm{Xin}$ & $\begin{array}{l}\text { Herba Asari (North) }{ }^{(2 x)} / \text { Herba } \\
\text { Asari/Herba Asari Cum Radice }\end{array}$ & 1 & 10 & $\begin{array}{l}<\text { LOQ- } \\
31\end{array}$ & 9 & ND & \\
\hline $\begin{array}{l}\mathrm{Xi} \text { Xin (in } \\
\text { formula) }\end{array}$ & Dang Gui Si Ni Tang & Tangkuei \& Jujube & & 1 & $<\mathrm{LOQ}$ & & ND & \\
\hline \multirow[t]{2}{*}{ Wei Ling Xian } & Wei Ling Xian & $\begin{array}{l}\text { Clematis/ Radix Clematidis/ } \\
\text { Radix Clematidis Chinensis }\end{array}$ & 10 & & ND & & ND & \\
\hline & $-/-$ & Clematidis/Radix Clematidis & 5 & & ND & & ND & \\
\hline $\begin{array}{l}\text { Wei Ling Xian (in } \\
\text { formula) }\end{array}$ & Shu Jing Huo Xue Tang & Clematis \& Peony Comb & 1 & & ND & & ND & \\
\hline Ba Yue Zha & Ba Yue Zha & Fructus Akebiae & 2 & & ND & & ND & \\
\hline
\end{tabular}

The combinations of Chinese common names and Latin pharmaceutical names were copied from the label or provided by the vendor. No translations of Chinese names into Latin pharmaceutical names were added by the authors. Dashes indicate that a name was not present on the label or that the vendor could not specify the name. Chinese common names with small differences which were deemed alternative names were grouped

$L O Q$ limit of quantification, $N D$ not detected

icants [12]. The pyrrolizidine alkaloid containing herbs are $\mathrm{Zi}$ Cao (Arnebia euchroma and Lithospermum erythrorhizon), Kuang Dong Hua (Tussilago farfara) and Pei Lan (Eupatorium fortunei) [35]. Altogether, based on our results it can be concluded that especially single herb THPs under the Chinese common names of Mu Tong, Fang Ji, Tian Xian 
Table 6 Randomly sampled Chinese THPs negative for AAs as determined with LC-MS

Chinese common name, as labeled

Ba Zhen Wan

Ba Zheng Ke Li

Bai Hua She She Cao

Bai Qui Feng

Bao He Wan

Bi Xie

Bian Dou

Bing Lang

Cang Zhu

Che Qian Cao ${ }^{(2 x)}$

Chuan Wu/Chuang Mu/-

Da Huang $(2 \mathrm{x})$

Dang Gui

Dao Chi Pian

Ding Chuan Wan

Du Zhong Bu Yao Granules

Fan Xie Ye

Fang Feng

Fu Ling ${ }^{(3 x)} /-(2 x)$

Fu Ping

Gui Fu Di Huang Wan

Guo Teng

He Shou Wu ${ }^{(2 x)} /-$

Hong Hua

Huai Hua

Huang Jing

Huang Lian Tang Granules

Huo Xiang Zheng Qi Wan

Je Yiao Teng

Jian Pi Wan

Jinkuishenqiwan

$\mathrm{Ku}$ Shen Pian

Kuan Dong Hua ${ }^{(3 \mathrm{x})} /$ - $^{(2 \mathrm{x})} /$ Dong Hua

Lei Gong Teng

Li Zhong Ke Li

Ma Chi Xian

Mai Wei Di Huang Wan

Mi Niao Ning Ke Li

Mi niao ning ke li

Qing Fei Ping Chuan Tang Granules

Qing Qi Hua Tan Pian

Quang Huo Rhizoma

Ren Shen Ye

Sang Ju Yin Ke Li

Sang Zhi

Shenzhi Jiaonang

Shu Gan Wan

Shugawan

$\mathrm{Su} \mathrm{Mu}$

Te Xiao Yao Tong Ling

Tiang Huang

$\mathrm{Wu}$ ji bai fe

$\mathrm{Wu}$ ji bai feng wan

Wu Yao

Xi Zhi Ren

Xia Sang Ju Chong Ji
Latin pharmaceutical name, as labeled

Octo Form granules

Herba Hedyoti Diffusae

$-$

Rhizoma Dioscorea Hypoglaucae

Semen Lablab Album

Rhizoma Atracttylodi

Plantago Asiatica/Herba Plantagaginis

R. A. Carmichaeli ${ }^{(2 x)} /$ Aconit Carmichaeli Preparata

Chinese Angelica

Scarlet Form

$-$

Folium Sennae

Radix Sapashnikovae S

Sclerotium Poriae Cocos $(5 x)$

$-$

Rumulus Unicare Cum Uncis

Radix Polygoni Multiflori ${ }^{3 x}$ )

Flos Carthami Tinctorii

Flos Sophorae

Rhizoma Polygonati, Polygonum officinale

$-$

Caulis Polygoni Multiflori

$-$

Flos Tussilagi Farfarae ${ }^{(6 x)}$

Herba Polygoni Perfoliali

Midrif Form granules

Herba Portulacae

$-$

Radix Notop Tergii

Folium Ginseng

Chrysanth Form

$-$

Lignum Sappan

$-$

R. L. Strychnifoliae

Black Cardamom 
Table 6 (continued)

\begin{tabular}{|c|c|}
\hline Chinese common name, as labeled & Latin pharmaceutical name, as labeled \\
\hline Xiang Yuan & Flos C. Mediae \\
\hline Xiao Feng Ke Li & Lay Wind Form \\
\hline Xiao Ji & - \\
\hline Xiao Yao San & Tangkuei \& Bupleurum Formula \\
\hline Ya Dan Zi & Fructus Brucae Jav \\
\hline Yan Fu Mu & - \\
\hline Yang Xue Sheng Fa Jiao Nang & - \\
\hline Ye Jiae Tang & - \\
\hline Yin Chen & Herba Artemisiae Scopariae ${ }^{(2 x)}$ \\
\hline Yu Mi Xu & Stigma Maydis \\
\hline $\mathrm{Yu} \mathrm{Zhu}{ }^{(2 \mathrm{x})} /-$ & Rhizoma Polygonati/ Polygonati Odorati $(2 \mathrm{x})$ \\
\hline Ze Lan/- & Herba Lycopi ${ }^{(2 x)}$ \\
\hline $\mathrm{Zi} \mathrm{Cao}$ & Radix Arnebiae S. Lithosspermi ${ }^{(2 x)}$ \\
\hline $\mathrm{Zi} \mathrm{Cao}{ }^{(2 x)}$ & Radix Arnebiae ${ }^{(2 x)}$ \\
\hline Zi Hua Di Ding & Herba Violae \\
\hline Zuo Gui Wan & - \\
\hline- & Aconite Ginseng \& Ginger Combination \\
\hline- & Artemisia Scoparia \\
\hline- & Astragalus extract tablets \\
\hline- & Beautifying and slimming tea \\
\hline- & Bupleurum \& Dragon Bone Combination \\
\hline- & Flos Chrysanthemi ${ }^{(3 x)}$ \\
\hline- & Herba Artemisiae Annuae \\
\hline- & Herba Eupatorii Fortunei ${ }^{(2 x)}$ \\
\hline- & Herba Lobeliae Chinensis Cum Radice ${ }^{(2 x)}$ \\
\hline- & Plantaginia Semen extract \\
\hline- & Radix Phytolaccae \\
\hline- & Radix Pulsatilae Chinenses \\
\hline- & Radix Rubiae \\
\hline- & Rehmannia Eight Formulas \\
\hline- & Rhizoma Dryopteria Crassihizomae ${ }^{(2 x)}$ \\
\hline- & Taraxaci Herbs \\
\hline
\end{tabular}

Teng and Xi Xin could be contaminated with AAs. Besides single-herb THPs, certain multi-ingredient THPs can be at risk of adulteration as well (Table 5).

In the following paragraphs we will expand on the possible reasons for contamination of these particular THPs with AAs.

\section{Mu Tong}

We found AAs in seven out of 14 single-herb THPs with the pharmaceutical names Caulis Akebia or Caulis Clematis armandii or $\mathrm{Mu}$ Tong with or without the prefixes "Guan" or "Chuan." The common Chinese name Guan $\mathrm{Mu}$ Tong exclusively refers to the stem of $A$. manshuriensis [23] and two samples exclusively labeled as Guang $\mathrm{Mu}$ Tong [sic] contained an AA I level of 919 and $1,000 \mathrm{mg} / \mathrm{kg}$ or higher and the AA II contents were 82 and more than $100 \mathrm{mg} / \mathrm{kg}$, respectively. Hashimoto et al. [3] reported AA I contents of
A. manshuriensis (Kan-mokutsu in Japanese) ranging between 0.169 and $0.882 \%$, which is more than $1.5-8.8$ times higher than the levels we found. Trujillo et al. [30] reported an AA content of $2,830 \mathrm{mg} / \mathrm{kg}$ in a sample of $A$. manshuriensis stem. In our study another sample, verbally indicated to be "Guang $\mathrm{Mu}$ Tong" [sic] but labeled as Caulis Clematidis Armandii contained no AAs. The stem of C. armandii is called Chuan $\mathrm{Mu}$ Tong, however. We therefore conclude that the prefix "Guang" was mistaken for "Chuan," which would explain the absence of AAs. A further five samples labeled as Caulis Clematidis Armandii with or without $\mathrm{Mu}$ Tong did, as expected, not contain AAs. Of the positive samples, three were labeled as a Chuan Mu Tong and Caulis Akebia (stem of A. quinata or A. trifoliata), which to our knowledge is not a common combination. The common Chinese name of Caulis Akebia is Bai $\mathrm{Mu}$ Tong and not Chuan $\mathrm{Mu}$ Tong, which refers to the stem of $C$. armandii and $C$. montana [1, 23]. 
Furthermore, two THPs labeled as Mu Tong, one of which also labeled as Caulis Akebiae, contained high levels of both AA I and AA II. These findings strongly underline the fact that the nomenclature of THPs cannot to be relied upon in some cases. Also the fact that AAs were found in half of the $\mathrm{Mu}$ Tong samples indicates that this group of THPs needs constant monitoring. Bensky and Gamble [1] noted that in premodern China Akebia was used as Mu Tong but that at present $A$. manshuriensis is used most often.

The formula Long Dan Xie Gan Wan is included in the FDA list of potential AA-containing herbs and formulas [24]. The suffix "Wan" in the formula name refers to pill in Pin Yin [1]. The formula Long Dan Xie Gan Tang is reported to contain ten ingredients among which is Caulis $\mathrm{Mu}$ Tong [25], for which either A. manshuriensis or the known Akebia spp. and Clematis spp. can be used [1]. In our study we sampled Long Dan Xie Gan and related products with the suffixes "Pian" (tablet) and "Tang" (decoction) [1, 25] and found 41-59 mg/kg AA I in three of eight related samples. Health authorities such as Health Canada and the MHRA in the UK issued warnings against the use of this product in 2002 and 2003 [15, 16]. Alternative names are "Quell fire" and "Lung Tan Xie Gan pills" [36]. Quell fire tablets were taken of the market in 2000 and reformulated as requested by the FDA after the detection of AAs [37]. We found no AAs in one sample of Quell fire which had a different lot number and expiry date than the earlier recalled lots [37]. A case of end-stage renal failure and recurrent carcinomas in the bladder due to the 5year use of Longdan Xieganwan manufactured in China was reported in the UK [38]. The formulas Dao Chi San and Xiao Feng San were each sampled once and were found to be negative for AAs in our study. Both formulas contain Caulis $\mathrm{Mu}$ Tong but some of the classical sources describe Xiao Feng San without this herb however [25]. Xiao Feng San has been found to contain AAs in Australia [18]. The FDA included the formula Dao Chi Wan in the listing of THPs suspected to contain AAs [24]. This formula has probably the same composition as Dao Chi San except that the latter is a powder (San) instead of a pill (Wan) [25]. It is prudent to include the formulas Long Dan Xie Gan, Dao Chi San and Xiao Feng San in a sampling protocol because of the possible inclusion of A. manshuriensis.

\section{Mu Xiang and Tian Xian Teng}

No AAs were detected in four related $\mathrm{Mu}$ Xiang samples. This common name can refer to the roots of $A$. debilis, Aucklandii lappa, Saussurea lappa, Inula helenium, I. racemosa and Vladimiria souliei [23]. Qing Mu Xian exclusively refers to the root of $A$. debilis (Table 1) but a sample of this THP contained contrary to expectations no AAs. Although our samples of Mu Xian, Guang Mu Xian (the root of S. lappa) and Vladimiria (Chuan Mu Xian is the root of $V$. souliei [1]) could potentially be substituted by A. debilis we did not detect AAs. This is in agreement with information from the European Agency for the Evaluation of Medicinal Products [23]. More research is needed however to evaluate the likelihood of this particular substitution. Another single-herb THP originating from an Aristolochia sp. is Tian Xian Teng, which according to the Chinese Pharmacopoeia is Herba Aristolochiae derived from A. contorta and A. debilis. A sample of this THP was found to contain $74 \mathrm{mg} / \mathrm{kg}$ of AA I and $33 \mathrm{mg} / \mathrm{kg}$ of AA II. Although Tian Xian Teng was sampled once and might not be very common on the market it should be included in sampling protocols.

\section{Fang $J i$}

In TCM the common name Fang Ji generally refers to several different herbs, namely, Cocculus trilobus, Cocculus orbiculatus, S. tetrandra and A. fangchi. Guang Fang Ji refers exclusively to A. fangchi and is the only of these species to contain AAs $[1,23]$. We sampled one THP verbally indicated as Guang Fang Ji which contained no AA however. A low concentration of AA was detected in a sample labeled as Fanji and Sclerotium Poriae Cocos. In a THP called Niu Ru Shiu, unknown to us, but also labeled as Cocculi Sarm. Rad. traces of AA I were detected. Obviously "Rad." stands for "Radix" and in the FDA list the herbs Cocculus sarmentosus and C. trilobus are included as alternative names for $C$. orbiculatus [24]. The root of $C$. orbiculatus as well as that of $C$. trilobus are called Mu Fang Ji in several sources [1, 39] and might therefore be substituted with Guang Fang Ji (A. fangchi) (Table 1) [24]. We found a relatively high AA I concentration of $523 \mathrm{mg} / \mathrm{kg}$ and $21 \mathrm{mg} / \mathrm{kg}$ of AA II in the multi-ingredient THP Mu Fang Ji Tang, which contains Radix Cocculi Trilobi (Mu Fang Ji) or Radix Stephania tetrandra (Han Fang Ji) according to some sources [40, 41] or Radix A. fangchi according to another source [42]. We found no AAs in three single-herb THP samples labeled as Radix Stephania tetrandra and/or Fang Ji. This does not indicate however that Fang Ji requires less attention. An AA-contaminated THP labeled as Stephania tetrandra was the cause of the Belgian AAN incident [20-22]. In a Swiss survey of AA I in commercial samples of slimming regimens consisting of Chinese plant mixtures four out of 42 tested positive. AA I was found in a sample Han Fang Ji declared to be $S$. tetrandra radix and traces were found in Han Fang Ji derived from Sinomenium acutum [28]. Both species are listed in the FDA list [24]. Another multi-ingredient THP analyzed by the Swiss researchers called Fang Ji Huang Qi Tang contained traces of AA I but a second sample did not [28]. We could not detect AAs either in Fang Ji Qi Tang, which could be a related formula. More attention should be 
focused on multi-ingredient THPs and the lists of these THPs potentially containing herbs with AAs should be expanded.

\section{Xi Xin}

We found low levels of AA I ranging between the LOQ and $31 \mathrm{mg} / \mathrm{kg}$ in ten out of 11 samples labeled as Xi Xin, Xi Xin (Bei) and Herba Asarum or Herba Asarum (North), Herba Asarum cum Radice samples. No AA II was found. In the multi-ingredient THP Dang Gui Si Ni Tang we found a small amount of AA I below the LOQ. This formula contains Xi Xin (Table 2) [25]. Xi Xin refers to Asarum sieboldii; the genus Asarum belongs to the Aristolochiaceae and could be expected to contain AAs. Hashimoto et al. [3] analyzed the Chinese Asarum spp. A. heterotropoides, A. sieboldii, A. splendens and A. himalaicum and A. forbesii and found only traces of AA I in A. splendens and A. himalaicum. Schaneberg et al. [4] found up to $370 \mathrm{mg} / \mathrm{kg}$ AA I but no AA II in the North American Asarum species A. canadense. Although the AA levels found in this study are low, batches of THPs containing Xi Xin should be routinely screened for AAs before they are brought on the market.

\section{Wei Ling Xian and Ba Yue Zha: likelihood of substitution by Aristolochia spp.}

In the FDA list Wei Ling Xian or the root of Clematis chinensis is included as a THP which may be adulterated with AA [24]. The Latin pharmaceutical name is Radix Clematidis and also refers to the roots of $C$. hexapetala and C. uncinata. Substitution of the roots of these herbs by, or contamination with, the stem of $C$. armandii or C. montana (Caulis Clematidis armandii or Chuan Mu Tong), which in turn can be replaced by the stem of A. manshuriensis (Guan $\mathrm{Mu}$ Tong), seems not likely because these THPs differ in appearance and moreover the roots of these last Clematis spp. are not reported to be in use for Wei Ling Xian [1, 43], which decreases the likelihood of this particular substitution as well. Another hypothetical option is confusion between Wei Ling Xian and Qing Mu Xian (the root of $A$. debilis) but we found no reports of this substitution. We analyzed ten samples of Wei Ling Xian/Radix Clematidis (Chinensis), five samples of Radix Clematidis and a formula containing this herb [25] but no AAs were found. Our results might indicate that Radix Clematidis or Wei Ling Xian is not likely to be replaced by Aristolochia spp. Nevertheless the FDA reported in 2001 the contamination of a $C$. chinensis extract with AAs [44]. More research into this substitution is therefore warranted.

Another herb where substitution by Aristolochia spp. seems unlikely is Fructus Akebiae or Ba Yue Zha. According to Bensky and Gamble [1] both names refer to the fruit of Akebia quinata and A. trifoliata. The fruit of these plants has also been reported to be referred to as Yu Zhi Zhi in the
Pharmacopoeia of the People's Republic of China and is entered as such in the FDA list [24, 45]. We found no AAs in two samples labeled as Fructus Akebiae and Ba Yue Zha. As the common Chinese name of Fructus Akebiae bears no resemblance to a common name of any Aristolochia sp., confusion would not seem likely. This has to be supported by additional results, however, before this specific THP may be considered for removal from the list of suspected materials. It might be possible that the fruits of $A$. debilis or A. contorta (Ma Dou Ling) and Fructus Akebiae are used interchangeably but we are not aware of reports of such a substitution.

\section{Exposure data}

The batches of $S$. tetrandra powder which were replaced by Aristolochia spp. in the Belgian AAN incident were reported to contain AA levels up to $1.56 \mathrm{~g} / \mathrm{kg}$ with a mean of $0.65 \mathrm{~g} / \mathrm{kg}$ [20]. Interestingly only in two of the 12 batches investigated was tetrandrine, the characteristic alkaloid of S. tetrandra, found and in one of these in combination with AAs. This would indicate that the rest of the batches consisted of $100 \%$ Aristolochia spp. replacing S. tetrandra [20]. It was estimated that the cumulative consumption of more than $200 \mathrm{~g}$ of these powders raised the risk of urothelial carcinoma [21]. This corresponds to a cumulative chronic intake of $130 \mathrm{mg}$ of AAs when using the mean AA content reported by Vanhaelen et al. [20]. We sampled a THP labeled as Chuan Mu Tong and C. Akebia on the Dutch market with an AA content of $2.1 \mathrm{~g} / \mathrm{kg}$ consisting of a natural mixture of $1.7 \mathrm{~g} / \mathrm{kg}$ of AA I and $0.44 \mathrm{~g} / \mathrm{kg}$ of AA II (Table 5). In a worst-case scenario $62 \mathrm{~g}$ of this THP with the highest AA content would supply more than $130 \mathrm{mg}$ of AAs, which, considering the Belgian data, could significantly raise the risk for cancer. According to Bensky and Gamble [1] the recommended dose of Mu Tong is 3-9 g, probably per day. The authors warn against overdose with reference to a case of acute renal failure following a dose of $60 \mathrm{~g}$. No limitation in the duration of use is given. When in our worst-case scenario the preparation with the highest AA content would be used following the highest dosing regimen, exposure to more than $130 \mathrm{mg}$ of AAs would be achieved in 7 days assuming that all AA is released from the matrix. In the Belgian cases of urothelial carcinoma the mean exposure duration was 15 months and generally end-stage renal failure occurred 3-85 months after cessation of the herbal regimen [21]. In Belgium the Fang Ji was consumed as a powder, which might have increased the exposure to AAs. In China, however, Chinese THPs are mostly used as decoctions, which might reduce the toxicity of Aristolochia spp. However, in Chinese literature two cases of acute renal failure after consumption of a decoction made of 70 and $175 \mathrm{~g}$ of Mu Tong, probably Guan Mu Tong, and four deaths of renal failure after consumption of decoctions of 50-120 g 
of $\mathrm{Mu}$ Tong were reported [46]. In the UK two cases were reported of end-stage renal failure following the use of $\mathrm{Mu}$ Tong containing AA I and AA II. Mu Tong was consumed by one patient as a tea for 6 years and the other patient used a preparation for 2 years in a undisclosed way [47].

\section{Conclusion}

Our finding that AAs were detected in 25 of 68 THP samples which could include AA-containing herbs indicates that several years after the ban in the Netherlands the risk of inadvertent exposure to AAs remains significant for those who use these particular THPs. In 1999 the MCA, now the MHRA, found AAs in $40 \%$ of the samples with Fang Ji and $\mathrm{Mu}$ Tong on the UK market [23]. Although the number of samples is relatively small, we found a similar percentage of samples containing AAs, namely, $37 \%$ of the suspected samples, which indicates that the situation has not improved since. In the UK the use of Mu Tong, Fang Ji, Ma Dou Ling or Qing Mu Xiang has been prohibited since 2001. Amongst these are also species such as $S$. tetrandra, Clematis spp. and Akebia spp. which do not belong to the Aristolochiaceae and in themselves do not contain AAs [15]. The Dutch Commodities Act "Herbal preparations" used to prohibit the sale of Magnolia officinalis and S. tetrandra as well. After a reevaluation of the literature it was concluded that these herbs in themselves pose little risk and subsequently the prohibition of these herbs was discontinued but it was recognized that a risk of substitution of $S$. tetrandra by A. fangchi remained [48].

Internationally the problem of AA-contaminated THPs still requires attention several years after measures by regulatory authorities in countries such as Great Britain, New Zeeland, Canada and Australia and the publicity generated by this [14-18]. The Belgian AAN tragedy clearly illustrates that contamination of THPs with AAs can have very serious consequences. Continued enforcement of the ban of AAs in the Netherlands will show if the problem of AA contamination of Chinese THPs is addressed more actively in the field of trade and if stricter regulatory measures are warranted. When identified, contaminated products will be removed from the Dutch market. The VWA will also in collaboration with customs direct its enforcement at the import of herbal material in order to prevent AA-containing THPs from entering the market. More research into possible contamination of THPs will help to safeguard the quality of Chinese THPs. As contamination is unnecessary TCM practitioners, manufacturers, vendors and importers of Chinese THPs should structurally direct efforts to the avoidance of AAs in THPs known to have the potential for contamination with AAs. Certification of THPs might aid to prevent the import of
AA-contaminated products but such a system needs close monitoring. It can be concluded that testing of the imported herbs for AA contamination is still essential.

\section{References}

1. Bensky D, Gamble A (1993) Chinese herbal medicine: materia medica.. Eastland, Seattle

2. Mix DB, Guinaudeau H, Shamma M (1982) J Nat Products 45:657-666

3. Hashimoto K, Higuchi M, Makino B, Sakakibara I, Kubo M, Komatsu Y, Maruno M, Okada M (1999) J Ethnopharmacol 64:185-189

4. Schaneberg BT, Applequist WL, Khan IA (2002) Pharmazie 57:686-689

5. Arlt VM, Stiborova M, Schmeiser HH (2002) Mutagenesis $17: 265-277$

6. Cosyns JP (2002) Facta Universitatis Med Biol 9:49-52

7. Debelle FD, Nortier JL, De Prez EG, Garbar CH, Vienne AR, Salmon IJ, Deschodt-Lanckman MM, Vanherweghem JL (2002) J Am Soc Nephrol 13:431-436

8. Lebeau C, Debelle FD, Arlt VM, Pozdzik A, De Prez EG, Phillips DH, Deschodt-Lanckman MM, Vanherweghem JL, Nortier JL (2005) Nephrol Dial Transplant 20:2321-2332

9. Hranjec T, Kovac A, Kos J, Mao W, Chen JJ, Grollman AP, Jelakovic B (2005) Croat Med J 46:116-125

10. IARC (2002) IARC monographs on the evaluation of carcinogenic risks to humans. Some traditional herbal medicines, some mycotoxins, naphthalene and styrene. Summary of data reported and evaluation. Volume 82. IARC, Lyons. http://monographs.iarc. fr/ENG/Monographs/vol82/volume82.pdf

11. Stiborova M, Frei E, Sopko B, Sopkova K, Markova V, Lankova M, Kumstyrova T, Wiessler M, Schmeiser HH (2003) Carcinogenesis 24:1695-1703

12. Rietjens IMCM, Martena MJ, Boersma MG, Spiegelenberg W, Alink GM (2005) Mol Nutr Food Res 49:131-158

13. Martena MJ, van der Wielen JCA, Klerx WNM, de Groot HN, Rietjens IMCM (2006) Chem-Biol Interact 161:165-175

14. FDA (2001) FDA concerned about botanical products, including dietary supplements, containing aristolochic acid. US Food and Drug Administration. http://www.cfsan.fda.gov/ dms/ds-bot.html

15. MHRA (2003) Aristolochia-Xie Gan Wan, Long Dan Xie Gan Wan, Guan Xin Su He, Longdan Qiegan Wan (Lung Tan Xie Gan). The Medicines and Healthcare Products Regulatory Agency. http:// www.mhra.gov.uk/home/idcplg?IdcService=SS_GET_PAGE\& useSecondary $=$ true $\&$ ssDocName $=$ CON024018\&ss TargetNo deId $=664$

16. Health Canada (2002) Warning not to consume Longdan and Lung Tan Xie Gan products. Health Canada. http://www.hc-sc.gc. ca/ahc-asc/media/advisories-avis/2002/2002_38_e.html

17. TGA (2001) Aristolochia fact sheet. Therapeutic Goods Administration. http://www.tga.gov.au/docs/html/aristol.htm

18. Medsafe (2003) Herbal, traditional and complementary medicines. New Zealand Medicines and Medical Devices Safety Authority. http://www.medsafe.govt.nz/hot/Herbal/ChineseMed/ DocLtr.asp

19. Vanherweghem JL, Depierreux M, Tielemans C, Abramowicz D, Dratwa M, Jadoul M, Richard C, Vandervelde D, Verbeelen D, Vanhaelen-Fastre R, Vanhaelen M (1993) Lancet 341:387-391

20. Vanhaelen M, Vanhaelen-Fastre R, But P, Vanherweghem JL (1994) Lancet 343:174

21. Nortier JL, Martinez MC, Schmeiser HH, Arlt VM, Bieler CA, Petein M, Depierreux MF, De Pauw L, Abramowicz D, Vereerstraeten P, Vanherweghem JL (2000) N Engl J Med 342:1686-1692 
22. Muniz Martinez MC, Nortier J, Vereerstraeten $P$, Vanherweghem JL (2002) Nephrol Dial Transplant 17:408-412

23. EMEA (2005) Public statement on the risks associated with the use of herbal products containing Aristolochia species. EMEA, London. http://www.emea.europa.eu/pdfs/human/hmpc/ 13838105en.pdf

24. FDA (2001) Listing of botanical ingredients of concern. US Food and Drug Administration. http://www.cfsan.fda.gov/ dms/ds-bot2.html

25. Bensky D, Barolet R (1990) Chinese herbal medicine: formulas and strategies. Eastland, Seattle

26. Yan, X, Zhou J, Xie G, Milne GWA (ed) (1999) Traditional chinese medicines: molecular structures, natural sources and applications. Ashgate, Aldershot

27. MCA (2001) Traditional ethnic medicines: public health and compliance with medicines law. Medicines Control Agency. http://www.escop.com/bhma/bhma/frame.htm

28. Ioset JR, Raoelison GE, Hostettmann K (2003) Food Chem Toxicol 41:29-36

29. Flurer RA, Jones MB, Vela N, Ciolino LA, Wolnik KA (2000) Determination of aristolochic acid in traditional Chinese medicines and dietary supplements. USFDA Forensic Chemistry Center, Cincinnati. http://www.cfsan.fda.gov/ acrobat/lib4212.pdf

30. Trujillo WA, Sorenson WR, La Luzerne P, Austad JW, Sullivan D (2006) J AOAC Int 89:942-959

31. Zhao Z, Yuen JPS, Wu, J Yu T, Huang W (2006) Ann Acad Med Singapore 35:764-769

32. Liang Z, Jiang Z, Leung KSY, Chan CL, Zhao Z (2006) J Food Drug Anal 14:36-43

33. Johanns ES, van der Kolk LE, van Gemert HM, Sijben AE, Peters PW, de Vries I (2002) Ned Tijdschr Geneeskd 146:813-816

34. Chan TY (2002) Drug Saf 25:823-828

35. Swissmedic (2005) Schweiz Ärzteztg 86:821

36. East Earth Trade Winds (2007) Information on herbal preparations from China. http://www.eastearthtrade.com/productinfo.php
37. FDA (2000) East Earth Herb recalls Jade Pharmacy brand Meridian Circulation and Quell Fire because of possible health risk. US Food and Drug Administration. http://www.fda.gov/oc/ po/firmrecalls/eastearth11_00.html

38. Laing C, Hamour S, Sheaff M, Miller R, Woolfson R (2006) Lancet 368:338

39. Rootdown (2007) Herbs. http://www.scootdown.com/mu-fang-ji? HerbCategory $\mathrm{ID}=20$

40. TCM Assistant (2007) Formulas. http://www.tcmassistant.com/ search/index.asp

41. CCMP (2007) Stephania and ginseng decoction, Mu Fang Chi Tang, Mu Fang Ji Tang. Committee on Chinese Medicine and Pharmacy, Department of Health, Taiwan. http://www.ccmp.gov. tw/en/information/formula_detail.asp? detailno $=20 \&$ selno $=0 \&$ relno $=52 \& \mathrm{PageNo}=4$

42. Chang YS, Deng JS, Ku YR (2002) J Liq Chromatogr Relat Technol 25:961-975

43. Herbasin (2006) Herbasin Chinese herb database - Caulis Clematidis Armandii (Chuan Mu Tong). http://www.herbasin.com/ database/chuanmutong.htm

44. FDA (2001) Vital Nutrients recalls Joint Ease \& Verified Quality brand Joint Comfort complex because of adverse health risk associated with aristolochic acid. US Food and Drug Administration. http://www.fda.gov/oc/po/firmrecalls/vital5_01.html

45. Medicinal Plant Herbarium (2005) PPRC list of contents: single plant drugs. Medicinal Plant Herbarium at Southern Cross University. http://www.scu.edu.au/schools/ncm/herbarium/pprc.htm

46. But PP, Ma SC (1999) Lancet 354:1731-1732

47. Lord GM, Tagore R, Cook T, Gower P, Pusey CD (1999) Lancet 354:481-482

48. RIVM SIR (2004) Risicobeoordeling van 7 verboden kruiden. Rijksinstituut voor Volksgezondheid en Milieu, Centrum voor Stoffen en Integrale Risicoschatting, Bilthoven. http://www.vwa. nl/cdlpub/servlet/CDLServlet?p_file_id=10788 